\title{
Vitamin D Supplementation Changed Relationships, Not Levels of Metabolic-Hormonal Parameters in Autoimmune Thyroiditis
}

\author{
K. VONDRA ${ }^{1}$, R. BÍLEK ${ }^{1}$, P. MATUCHA ${ }^{1}$, M. SALÁTOVÁ ${ }^{1}$, M. VOSÁTKOVÁ ${ }^{1}$, \\ L. STÁRKA ${ }^{1}$, R. HAMPL ${ }^{1}$ \\ ${ }^{1}$ Institute of Endocrinology, Prague, Czech Republic
}

Received May 24, 2017

Accepted June 21, 2017

\section{Summary}

In women with chronic autoimmune thyroiditis and vitamin D deficiency we have found reference levels of relevant metabolichormonal parameters except for parathormone and total calcium. Three months supplementation with vitamin D (4300 IU/day, cholekalciferol) did not lead to significant changes of investigated hormonal parameters, while the levels of parathormone and calcium reached normal levels. However, a correlation analysis revealed marked changes in mutual relations. First, an inverse correlation of vitamin $D$ with parathormone, insulin secretion (C peptide, insulin) and its efficiency (HOMA IR) disappeared. Relationships of vitamin $D$ to hepatic insulin resistance (insulin/C peptide), to DHEA (both negative), and to DHEAS/DHEA ratio (positive) were newly found. Second, a positive correlation of CRP with insulin secretion remained, while its relation to insulin efficiency (HOMA IR, insulin/ $C$ peptide) was newly observed. Analogical positive correlations appeared also among anti TPO and insulinemia, insulin/C peptide, HOMA IR, and anti $\mathrm{Tg}$ to $\mathrm{C}$ peptide. A relationship of the CRP with anti TPO became significant (+). Third, out of glucose metabolism parameters only insulin/C peptide and glycemia did not correlate with vitamin D during its deficiency, while after supplementation insulin/ $C$ peptide alone correlated positively with both DHEAS and DHEA, and negatively with vitamin $\mathrm{D}$.

\section{Key words}

Vitamin D deficiency • Autoimmune thyroiditis • Supplementation of vitamin $D$

\section{Corresponding author}

K. Vondra, Institute of Endocrinology, Národní 8, 11600 Prague 1, Czech Republic. E-mail: kvondra@endo.cz

\section{Introduction}

Cross sectional, as well as observation studies point to association of vitamin D deficiency with autoimmune thyroiditis (AIT) and its forms. Our own experience fully agrees with the literature data (Vondra et al. 2015).

On the other hand results of prospective studies on a direct effect of vitamin $\mathrm{D}$ supplementation in humans are very limited. It is caused above all by the fact that the reason for association between increased occurrence of vitamin D deficiency, not only in the AIT, but also in other autoimmune diseases, has not yet been explained, though a causal relationship is not accepted by most of authors. In the context of available experimental data we may speculate about the role of insufficient immunomodulatory effect of low 1,25-(OH) ${ }_{2} \mathrm{D}_{3}$ concentrations in AIT, for instance due to lowered activity of both macrophages and dendritic cells, resulting in increased autoantigen presentation, or increased formation of pro-inflammatory cytokines and cell destructive Th1 lymphocytes. Considering the importance of vitamin D supplementation for the course of the AIT, the data from several intervention studies should be mentioned pointing to its beneficial effect. In the Polish study administration of vitamin D to non-lactating women with postpartum thyroiditis led to a decrease of anti TPO titers and this effect was more pronounced in women with vitamin D deficiency (Krysiak et al. 2016). In another open randomized control study in India threemonth supplementation of vitamin D to patients with AIT led to a decrease of anti TPO titre, this effect was significant primarily in patients with TSH levels below 
$10 \mathrm{mIU} / \mathrm{l}$ (Chaudhary et al. 2016).

In this work we attempted to gain more detailed information on a) the relationship of vitamin D deficiency to selected metabolic hormonal markers related to inflammation, insulin efficiency and bone metabolism in women with AIT, b) on the effects of vitamin D supplementation on relationships found during the deficiency.

\section{Patients}

Thirty women treated for a long time for chronic autoimmune thyroiditis (AIT) in the Institute of Endocrinology (Prague) were investigated for the levels of vitamin D, selected clinical and laboratory metabolichormonal parameters related to inflammation, glucoregulation, insulin efficacy and bone metabolism, before and after 3 months supplementation with vitamin D. All the patients were receiving a substitution therapy with thyroid hormones (levothyroxine). A selection criteria were at least one year lasting clinical and laboratory normal thyroid function, current vitamin $\mathrm{D}$ level below $50 \mathrm{nmol} / 1(<20 \mathrm{ng} / \mathrm{ml})$, absence of diabetes mellitus of both types, autoimmune or other serious diseases. Investigation was carried out during winter months between 2016/2017. The study was approved by the Ethical Committee of the Institute of Endocrinology, Prague.

The detailed characteristics of the patient group before starting of vitamin D substitution and after deficiency adjustment are shown in Table 1.

Vitamin D was administered in a form of cholecalciferol (Vigantol gtts) for 3 months in a dose 30 drops twice a week, corresponding in average to $4300 \mathrm{IU} /$ day. The vitamin D substitution did not lead to any complication for the whole period or to any side effects. Initial levels of $25(\mathrm{OH}) \mathrm{D}$ varied within the range 10-44 nmol/1, median $31 \mathrm{nmol} / 1$, while corrected values after 3 months were within the range $65-136 \mathrm{nmol} / \mathrm{l}$, median $57 \mathrm{nmol} / 1$.

\section{Methods}

Glucose was measured spectrophotometrically, using an enzymatic method with hexokinase (Cobas 6000, Roche, Mannheim, Germany). Total calcium was measured spectrophotometrically, using an NM-BAPTA method (5-nitro-5'-methyl-(1,2-bis(o-aminophenoxy) ethan-N,N,N',N'-tetraacetic acid) (Cobas 6000, Roche,
Mannheim, Germany).

Thyreotropin (TSH), free thyroxine fraction (fT4), free triiodothyronine (fT3), C peptide (Cp), insulin, parathyroid hormone $(\mathrm{PTH})$ and vitamin $\mathrm{D}(25(\mathrm{OH}) \mathrm{D}$ total $-25 \mathrm{OHD}_{2}+25 \mathrm{OHD}_{3}$ ) were measured by immunoanalytic ECLIA methods (Cobas 6000, Roche, Mannheim, Germany).

C reactive protein (CRP) was measured spectrophotometrically, using an immunoturbidimetric assay (Cobas 6000, Roche, Mannheim, Germany). Sex hormone binding globuline (SHBG) was measured by immunoradiometric assay (SHBG IRMA KIT) (Beckman Coulter Inc., Chaska, Minnesota, USA). Dehydroepiandrosterone (DHEA) and its sulfate (DHEAS) were measured by radioimunoassay (RIA DHEA) (Beckman Coulter, Prague, Czech Republic).

Thyroid auto-antibodies ( $\mathrm{T}-\mathrm{Ab}$ ) - antibodies to thyroid peroxidase (anti TPO) and antibodies to thyreoglobulin (anti Tg) were measured using an ELISA method (Aesku.Diagnostics, Wendelsheim, Germany) on an Immunomat BASE (Serion Immunologics, Germany).

\section{Statistics}

Descriptive statistics was performed by Microsoft Excel. The effect of vitamin D supplementation on the investigated parameters was performed by paired signed rank test.

Correlation of initial and corrected data was performed by Spearman correlation analysis.

\section{Results}

Initial values as well as those after vitamin D supplementation of clinical and laboratory parameters, expressed as medians and 25 th and 75 th quartiles are shown in Table 1. For comparison, there are also reference values for analyzed parameters as used in the Institute of Endocrinology, Prague (see www.endo.cz). It is evident from Table 1 that initial data are within the range of reference values and no significant changes occurred after vitamin D supplementation as proven by paired signed rank test. Changes of parathormone (PTH) and total calcium were the only exception, where the supplementation led to their full correction.

The results of Spearman correlation analysis of selected parameters before and after correction of vitamin $\mathrm{D}$ deficiency and their significancy are given in Tables 2 and 3. 
Table 1. Baseline and control parameters after vitamin D supplementation.

\begin{tabular}{|c|c|c|c|c|}
\hline \multirow{2}{*}{ Variable } & \multicolumn{2}{|c|}{ Median (quartiles) } & \multirow{2}{*}{ p-value } & \multirow{2}{*}{ Reference interval } \\
\hline & Baseline parameters & Control parameters & & \\
\hline Age (years) & \multicolumn{2}{|c|}{$41(31,48)$} & & \\
\hline$B M I\left(\mathrm{~kg} / \mathrm{m}^{2}\right)$ & $24.8(21.3,28.5)$ & $24.7(21.7,28.5)$ & n.s. & $18.5-25.0$ \\
\hline TSH (mIU/l) & $2.80(1.59,3.83)$ & $2.81(1.62,3.58)$ & n.s. & $0.27-4.20$ \\
\hline fT4 (pmol/l) & $15.8(14.2,17.4)$ & $16.8(14.6,17.8)$ & n.s. & $12.0-22.0$ \\
\hline fT3 (pmol/l) & $4.52(4.28,4.82)$ & $4.58(4.32,4.92)$ & n.s. & $3.10-6.80$ \\
\hline anti TPO (IU/ml) & $178(35.0,371.0)$ & $230(32.0,352.0)$ & n.s. & $<40.0$ \\
\hline anti $\operatorname{Tg}(I U / m l)$ & $29.0(6.9,126.0)$ & $31.0(10.0,90.0)$ & n.s. & $<120.0$ \\
\hline$C R P(m g / l)$ & $1.10(0.45,2.65)$ & $1.05(0.60,2.27)$ & n.s. & $0.0-5.0$ \\
\hline C peptide (nmol/l) & $0.65(0.56,0.80)$ & $0.71(0.56,0.93)$ & n.s. & $0.30-1.47$ \\
\hline Insulin $(m I U / l)$ & $8.80(6.75,11.2)$ & $8.9(6.85,12.4)$ & n.s. & $2.60-24.90$ \\
\hline Glucose (mmol/l) & $5.10(4.80,5.30)$ & $5.05(4.80,5.30)$ & n.s. & $3.90-5.60$ \\
\hline HOMA IR & $1.9(1.38,2.64)$ & $1.93(1.51,2.46)$ & n.s. & $x-x$ \\
\hline$P T H(n g / l)$ & $50.3(41.9,61.3)$ & $43.9(36.8,52.0)$ & $* * *$ & $15.0-65.0$ \\
\hline Calcium total (mmol/l) & $2.40(2.28,2.43)$ & $2.43(2.40,2.49)$ & $* *$ & $2.15-2.55$ \\
\hline SHBG $(\mathrm{nmol} / \mathrm{l})$ & $50.4(37.1,93.3)$ & $59.0(31.0,102.1)$ & n.s. & $43.0-95.0$ \\
\hline DHEA (nmol/l) & $22.7(17.3,32.3)$ & $19.9(10.8,29.6)$ & n.s. & $4.3-61.0$ \\
\hline DHEAS (umol/l) & $3.54(1.97,4.66)$ & $3.52(2.04,4.50)$ & n.s. & $1.80-7.20$ \\
\hline Vitamin D (nmol/l) & $30.9(22.5,38.5)$ & $86.4(57.5,98.0)$ & $* * *$ & $75.0-200.0$ \\
\hline
\end{tabular}

BMI - body mass index, TSH - thyrotrophic hormone, fT4 - free thyroxine fraction, fT3 - free triiodothyronine fraction, anti TPO - antithyroid peroxidase, anti $\mathrm{Tg}$ - anti-thyroglobulin, CRP - C-reactive protein, PTH - parathyroid hormone, HOMA IR - insulin resistance index, SHBG - sex hormone-binding globulin, DHEA - dehydroepiandrosterone, DHEAS - dehydroepiandrosterone sulfate. MannWhitney test was used for evaluation of between group differences: $* * P<0.01$, and $* * * P<0.001$, n.s. - not significant.

A correlation analysis revealed marked changes in mutual relations after supplementation as follows:

a) An inverse correlation(s) of vitamin $\mathrm{D}$ with PTH, insulin secretion (C peptide, insulin) and its efficiency ((HOMA IR) after supplementation no more were proven. Newly found relationships of vitamin $D$ to hepatic insulin resistance (insulin/C peptide), to DHEA (both negative), and to DHEAS/DHEA ratio (positive).

b) A positive correlation of CRP with insulin secretion remained, while newly recorded was its relation to insulin efficiency ((HOMA IR, insulin/C peptide). Analogical positive correlations appeared also among anti TPO and insulinemia, insulin/C peptide, HOMA IR, and anti Tgl to $\mathrm{C}$ peptide. A relationship of the CRP with anti TPO became significant $(+)$.

c) As parameters of glucose metabolism concern, only insulin/C peptide and glycemia did not correlate with vitamin $\mathrm{D}$ during its deficiency; while after supplementation insulin/C peptide alone correlated positively with both DHEAS and DHEA, and negatively with vitamin D. d) Inversion relationships of insulin, HOMA IR and insulin/C peptide respectively to sex hormonebinding globulin remained unchanged after supplementation.

\section{Discussion}

In our work we attempted to gain more detailed information on the relationship of the vitamin D deficiency before and after its correction with selected metabolic-hormonal laboratory parameters related to inflammation, glucoregulation, adrenal androgens, and bone metabolism in women with most frequent autoimmune endocrinopathy, namely AIT. The results brought a number of new insights on relationships between the degree of vitamin D deficiency and investigated parameters. In the discussion we tried to comment the results in the context of recent, often scarce knowledge. 


\begin{tabular}{|c|c|c|c|c|c|c|c|c|c|c|c|c|c|c|}
\hline ט. & 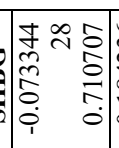 & $\mid \begin{array}{cc}\pi & 0 \\
0 & \infty \\
0 & 0 \\
0 & 3 \\
0 & 0\end{array}$ & 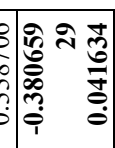 & 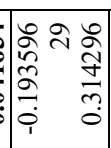 & 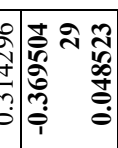 & $\mid$ & 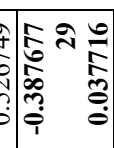 & 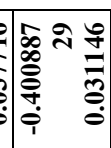 & 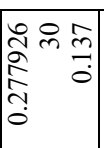 & $\left|\begin{array}{cc}0 \\
0 & 0 \\
0 & 0 \\
0 & 0 \\
0 & 0\end{array}\right|$ & $\begin{array}{cc}3 \\
6 & 5 \\
\vdots & 0 \\
1 & 0\end{array}$ & 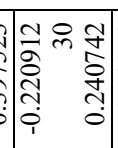 & 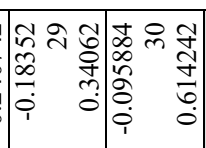 & \\
\hline & 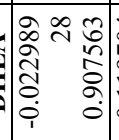 & 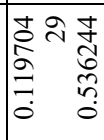 & 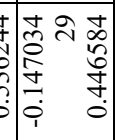 & 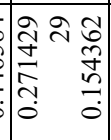 & 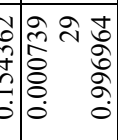 & 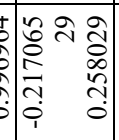 & 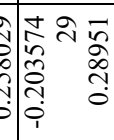 & 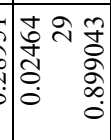 & 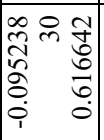 & $\mid \begin{array}{cc}0 & 0 \\
0 & 0 \\
0 & 0 \\
0 & 0 \\
0 & 0\end{array}$ & & 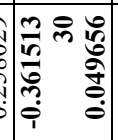 & 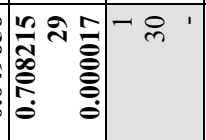 & 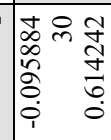 \\
\hline 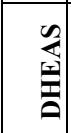 & 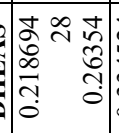 & 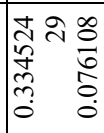 & 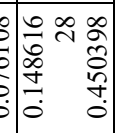 & 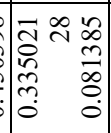 & 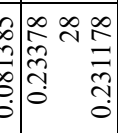 & 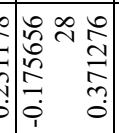 & 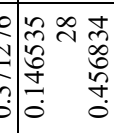 & 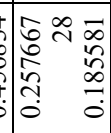 & 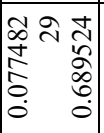 & 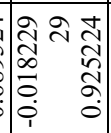 & 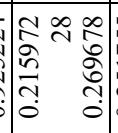 & 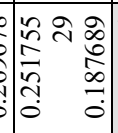 & 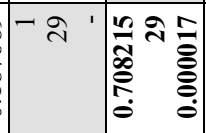 & $\begin{array}{l}0 \\
\infty \\
\infty \\
\infty\end{array}$ \\
\hline & 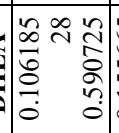 & 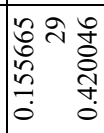 & 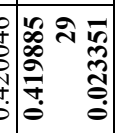 & 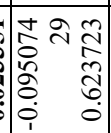 & 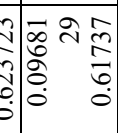 & $\hat{c}$ & 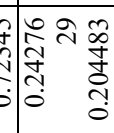 & 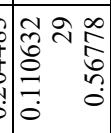 & $\mid$ & 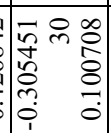 & . & i & 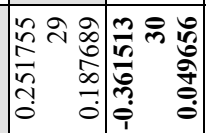 & 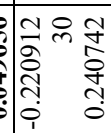 \\
\hline & 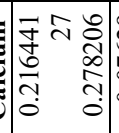 & & 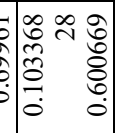 & 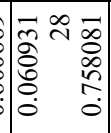 & 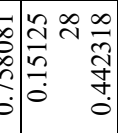 & 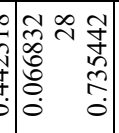 & 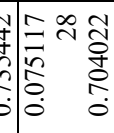 & 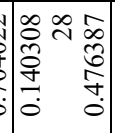 & 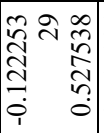 & 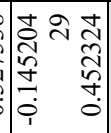 & & 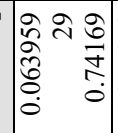 & 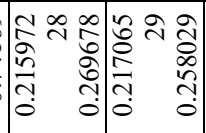 & 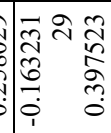 \\
\hline & 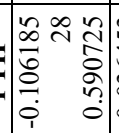 & 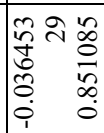 & 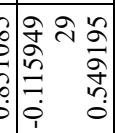 & 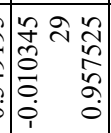 & 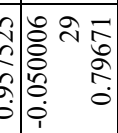 & 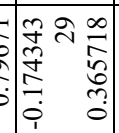 & 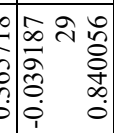 & 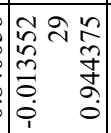 & 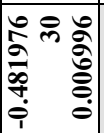 & & 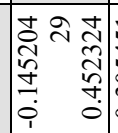 & 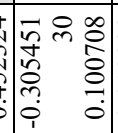 & 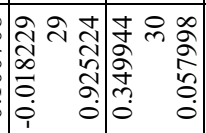 & 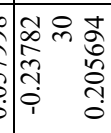 \\
\hline & 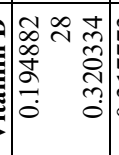 & 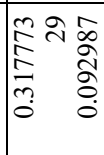 & 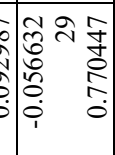 & 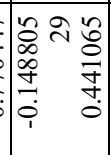 & 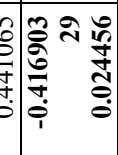 & $\begin{array}{lll}0 \\
f\end{array}$ & $\begin{array}{ll} \\
\hat{0}\end{array}$ & 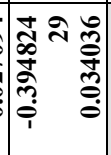 & & 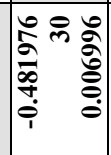 & 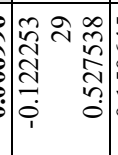 & 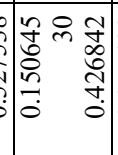 & 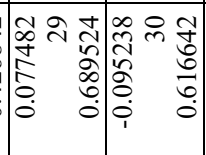 & 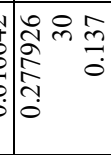 \\
\hline & 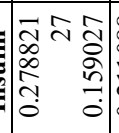 & 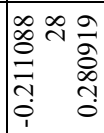 & 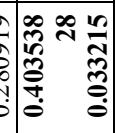 & 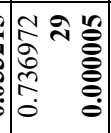 & $=$ & 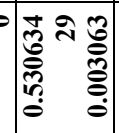 & 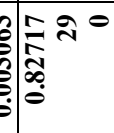 & & 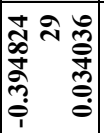 & 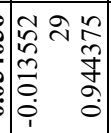 & $\frac{0}{0}$ & 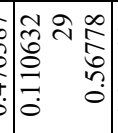 & 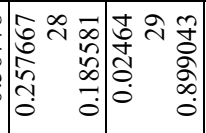 & 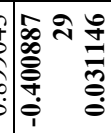 \\
\hline & 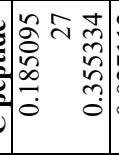 & 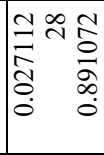 & 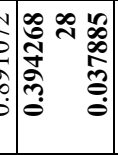 & $\left|\begin{array}{ccc}\hat{n} & 2 & 0 \\
0 & 2 & 0 \\
0 & 0 \\
& 0 \\
0 & 0 \\
0 & 0\end{array}\right|$ & 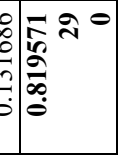 & 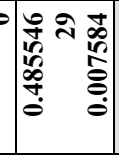 & 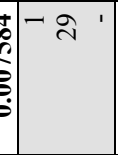 & 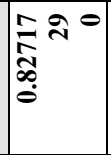 & 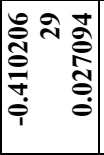 & 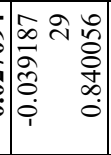 & 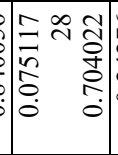 & 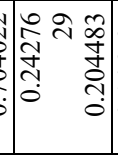 & 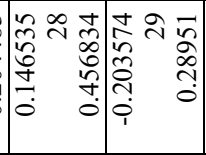 & 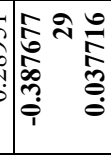 \\
\hline & 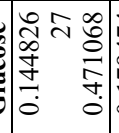 & 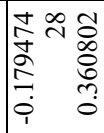 & 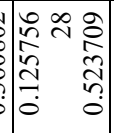 & $\begin{array}{l}\infty \\
0 \\
0 \\
2 \\
\vdots \\
0 \\
0\end{array}$ & 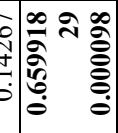 & & 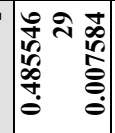 & 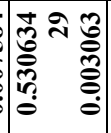 & 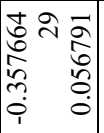 & 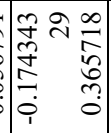 & {$\left[\begin{array}{cc}\infty & 1 \\
0 & 0 \\
0 & 0 \\
0 & 0\end{array}\right.$} & 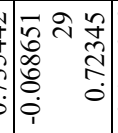 & 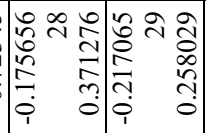 & 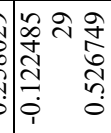 \\
\hline 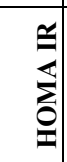 & 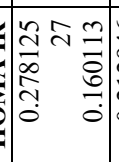 & 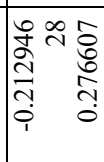 & 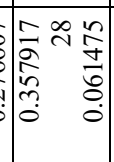 & 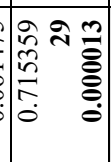 & & . & 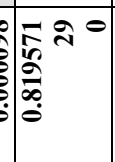 & 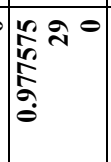 & 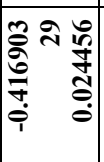 & 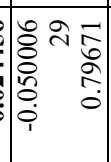 & 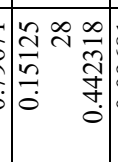 & 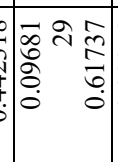 & 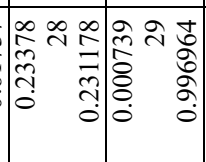 & 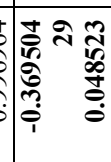 \\
\hline & 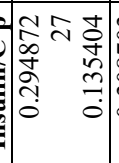 & 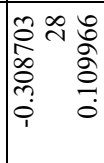 & 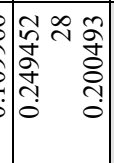 & & 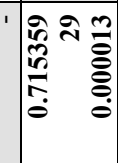 & 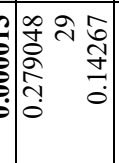 & 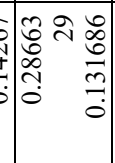 & 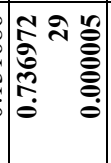 & 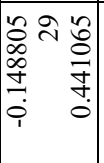 & 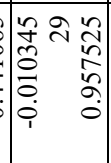 & \begin{tabular}{l}
$\prod^{\infty}$ \\
\hdashline \\
0 \\
0
\end{tabular} & 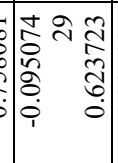 & 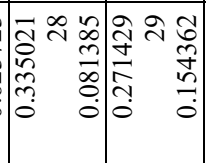 & $\begin{array}{l}0 \\
\vdots \\
2 \\
2 \\
2 \\
0 \\
0 \\
0\end{array}$ \\
\hline & 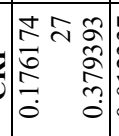 & 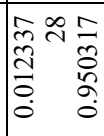 & & 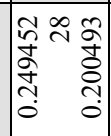 & 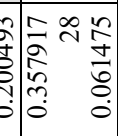 & 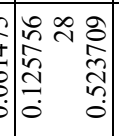 & 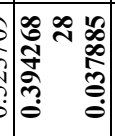 & 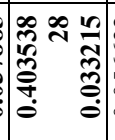 & 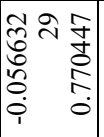 & 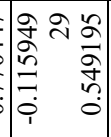 & 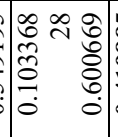 & 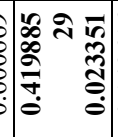 & 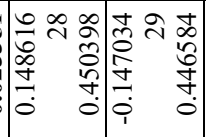 & 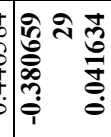 \\
\hline & 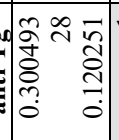 & & 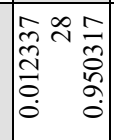 & $\mid \begin{array}{lll}0 & \infty & 0 \\
0 & 0 & 0 \\
0 & 0 \\
0 & 0 \\
0 & 0 \\
i & 0 \\
i & 0\end{array}$ & 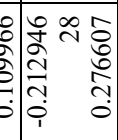 & 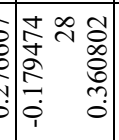 & 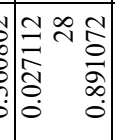 & 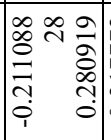 & 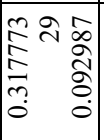 & 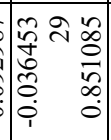 & 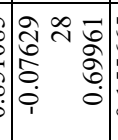 & 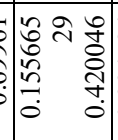 & 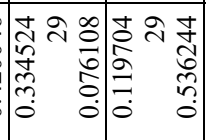 & 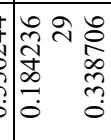 \\
\hline | & & 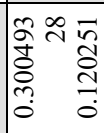 & 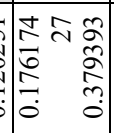 & 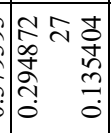 & 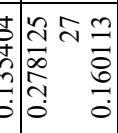 & 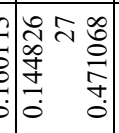 & 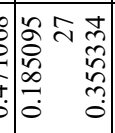 & 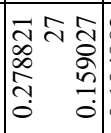 & 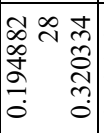 & $\left|\begin{array}{ccc}n & \infty & 0 \\
0 & 1 & 0 \\
0 & 0 \\
0 & 0 \\
0 & 0 \\
1 & 0 \\
1 & 0\end{array}\right|$ & 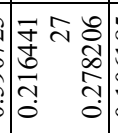 & 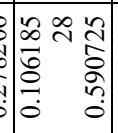 & 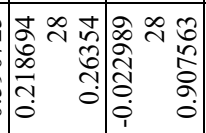 & 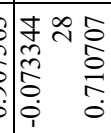 \\
\hline & : & & Uิ & Uे & לָ & 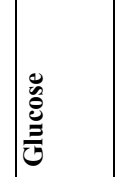 & Uू & : & & 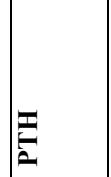 & 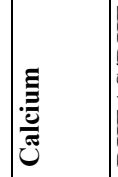 & $\bar{z}$ & 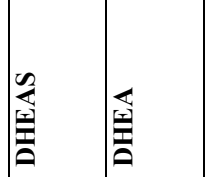 & 18 \\
\hline
\end{tabular}




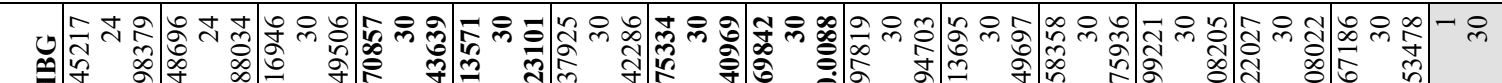

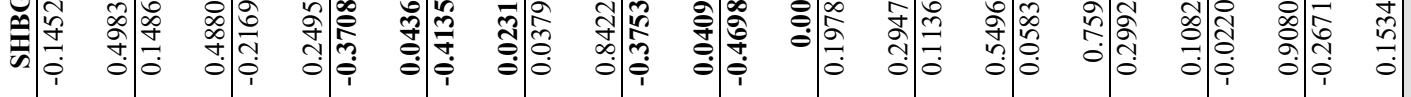

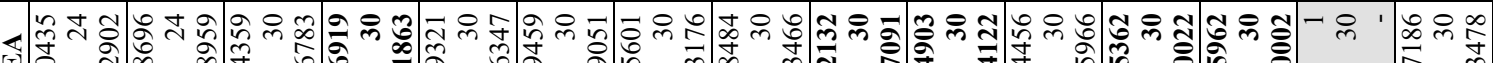

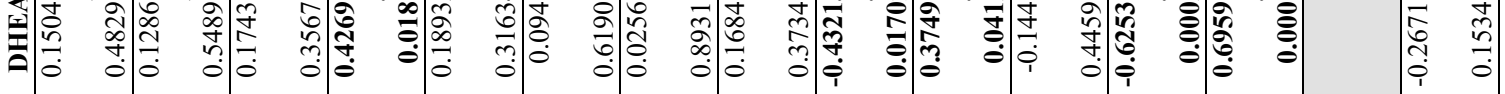

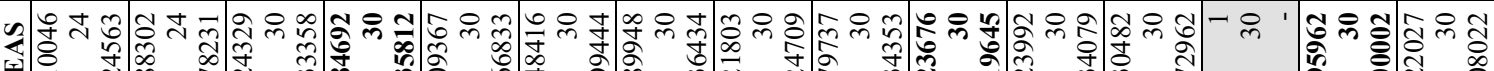

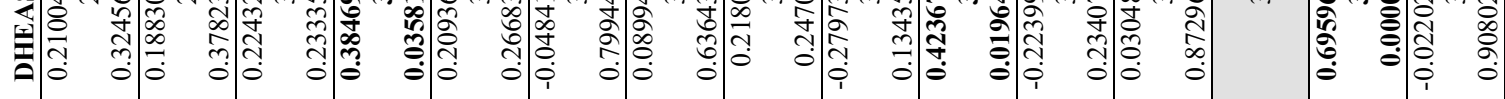

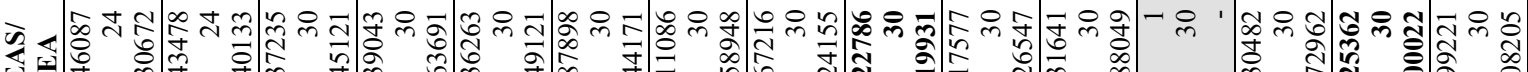

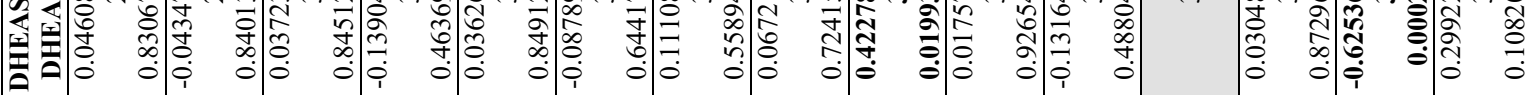

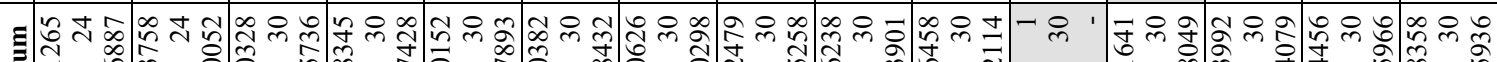

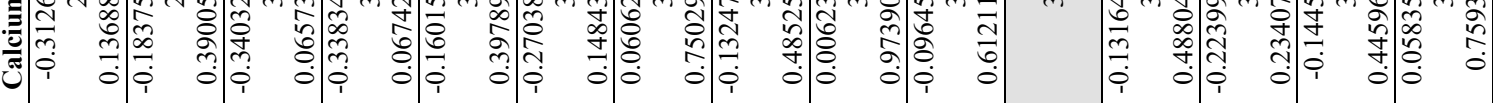

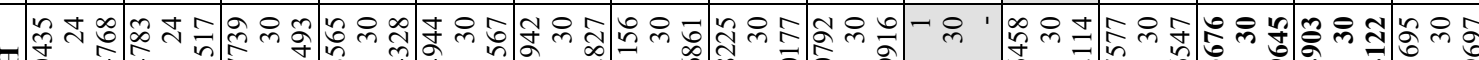

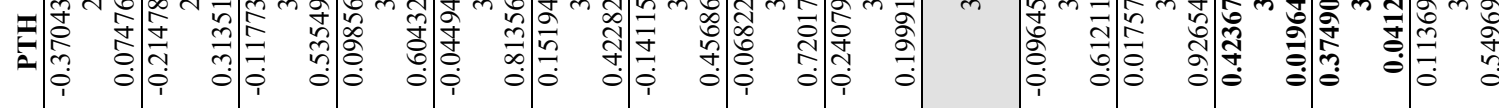

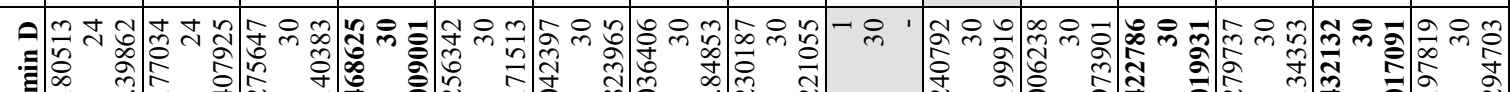

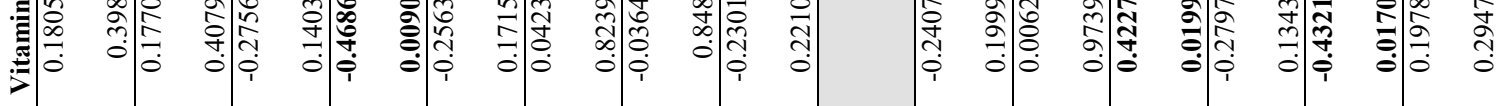

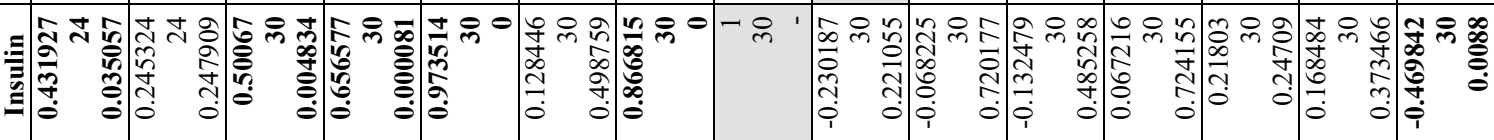

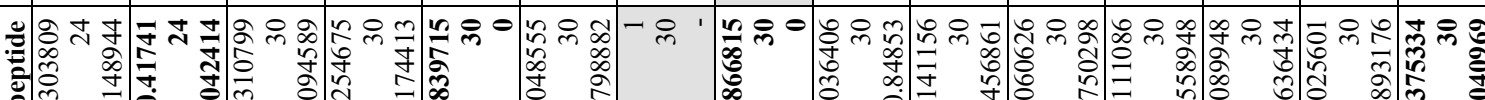

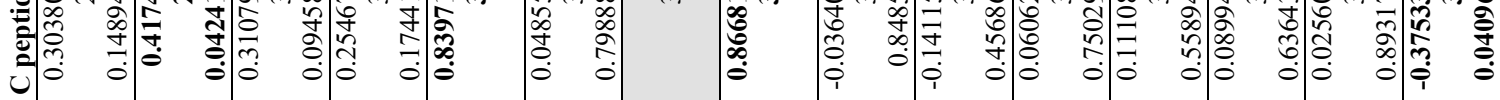

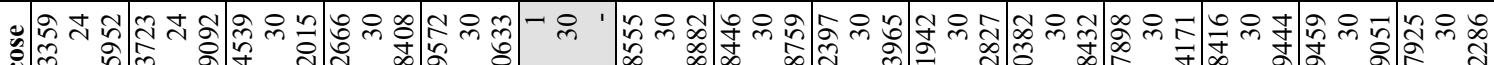

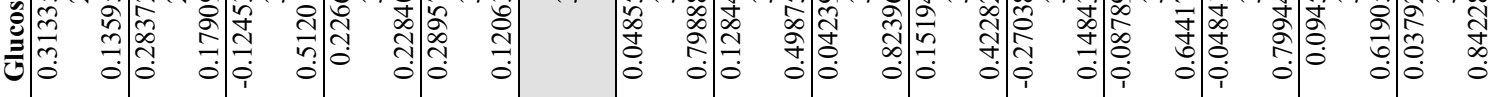

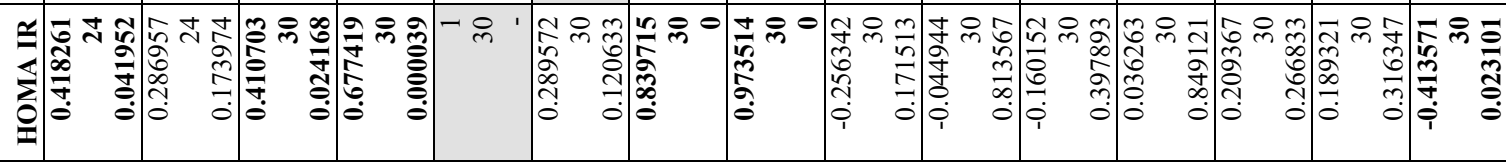

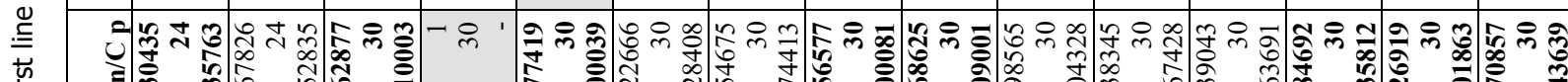
产

五

$\frac{\pi}{\frac{\pi}{0}}$

혼

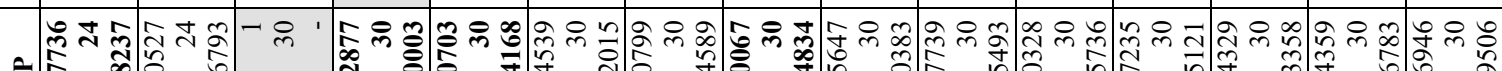

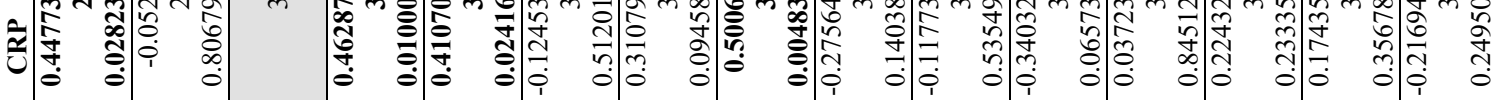
m

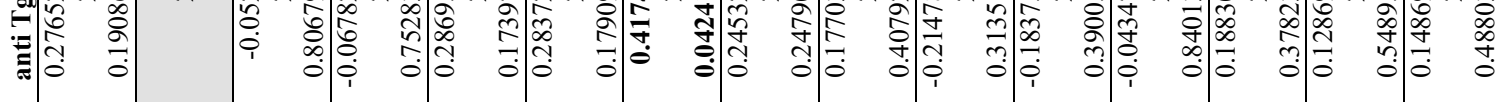

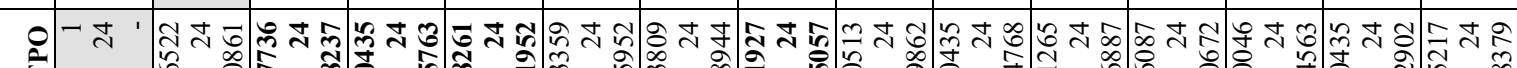

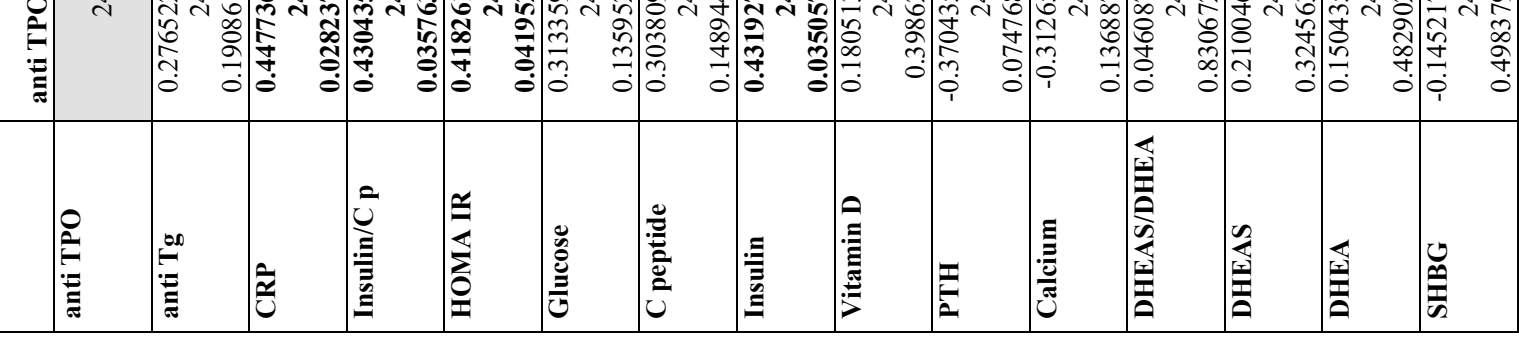


Baseline levels of thyroid hormones in investigated women laid in the normal range and thus at adequate substitution with thyroid hormones were not influenced by the vitamin D deficiency. The corrected values under the same thyroid substitution remained in the middle of the reference range. The correlation analysis during vitamin $\mathrm{D}$ deficiency revealed a positive relationship between the fT4/fT3 ratio and vitamin D level $(\mathrm{r}=0.4359, \mathrm{p}=0.016)$. We may speculate about a compensatory adaptation on a deep vitamin D deficiency. This is supported by the disappearance of the correlation between fT4/fT3 and vitamin D after its correction.

During vitamin D deficiency we observed a typical increase of parathormone levels, which in about one fourth of patients exceeded the upper limit of the reference range $(65.0 \mathrm{ng} / \mathrm{l})$. Elevated values corresponded to secondary hyperparathyroidism as a result of insufficient saturation with vitamin $D$. It was verified by a full correction of parathormone levels after vitamin D adjustment and could be further supported by a proven inverse relationship between vitamin $D$ and parathormone, which disappeared after supplementation. Basal values of total calcium laid within the reference range, their changes after correction of vitamin $\mathrm{D}$ deficiency were small though significant.

Cross matched studies dealing with association of vitamin D with inflammatory markers brought inconsistent results (Liefaard et al. 2015, Azizieh et al. 2016). This is true about studies on both healthy subjects as well as on patients with some inflammatory diseases (Kruit and Zanen 2016). Generally, it seems that an inverse correlation of vitamin D to CRP is more pronounced in severe inflammatory diseases (Cannell et al. 2015, Kruit and Zanen 2016). There are many factors provided in the literature responsible for heterogeneity in the response of inflammatory markers to vitamin $\mathrm{D}$ supplementation: most frequently it is basal $25(\mathrm{OH}) \mathrm{D}_{3}$ levels, but also initial value of inflammatory markers, the dose of supplemented vitamin D, its type (cholecalciferol or ergocalciferol) and the duration of vitamin D therapy. In these studies importance of higher basal values of inflammatory markers is emphasized, while in case of low values a further decrease after vitamin $\mathrm{D}$ levels correction is not probable.

Our findings on women under a long-term therapy with thyroxin substitution for AIT are in accordance with conclusions of the above cited studies. Low baseline values of CRP as well as T-Ab titres did not further decrease after correction of vitamin D deficiency, in agreement with experience of already mentioned authors. It was probably the reason that a decrease of an anti TPO titre did not occur, as described by Krysiak et al. (2016) or Chaudhary et al. (2016). Mutual positive correlations of CRP values with $\mathrm{T}-\mathrm{Ab}$ titres, recently mentioned in the literature (Taubner et al. 2014), could be proven here only in vitamin D-supplemented women, namely a positive correlation between CRP and anti TPO. A question arises whether a long-term administration of thyroid hormones to our investigated women participates at low values of $\mathrm{CRP}, \mathrm{T}-\mathrm{Ab}$ and their relationship to vitamin $\mathrm{D}$ levels. These relationships have not yet been systematically studied; only Krysiak et al. (2017) in a very recent study describes a decrease of thyroid autoimmunity by administration of vitamin D in levothyroxine-treated women with AIT and normal levels of vitamin D.

Sporadic literary data concerning the relationship of DHEA/S to AIT describe low levels of $\mathrm{DHEA} / \mathrm{S}$ in $\mathrm{T}-\mathrm{Ab}$ positive females, with an inverse relationship to anti TPO as well as to anti Tg (Ott et al. 2014). In our group the levels of DHEA/S (taking into account patient's age) laid in the middle of the reference range before as well as after correction of vitamin $\mathrm{D}$ deficiency.

In the state of vitamin D deficiency we have found only one significant positive correlation between CRP and the ratio DHEAS/DHEA reflecting the activity of steroids sulfotransferase enzyme. The meaning of this relationship may help explain experimental findings of Solerte et al. (2005). These authors have proven an association of dysregulation of natural killer (NK) cell cytotoxicity and NK-produced cytokines with thyroid autoimmunity, even during thyroxine therapy. In addition they have shown that a functional defect of NK cells can be reversed in vitro by incubation of NK cells with a dose-dependent treatment with DHEAS.

In the light of these experimental results the elevated values of the DHEAS/DHEA ratio at vitamin D deficient state associated with increasing levels of CRP could be considered a protection from higher activity and progression of the inflammation. This idea is supported by the fact that the correlation between DHEAS/DHEA to CRP disappeared after correction of vitamin D deficiency. On the other hand there were correlations of adrenal androgens (DHEA, DHEAS) to vitamin D, the same as described in healthy women. Zhao et al. (2017) described in a multiethnic cross sectional study with 
2929 women an inversion relationship of low vitamin D levels to DHEA, independent on the lifestyle and adiposity. An analogical correlation was found in our women with AIT after correction of vitamin D deficiency, an inverse relationship between vitamin D and DHEA was also manifested by a positive correlation with DHEAS/DHEA ratio. These relationships may be considered a consequence of DHEA sulfotransferase (SULT2A1) stimulation by vitamin D supplementation. Such an interpretation comes out of works emphasizing an important role of vitamin D for stimulation of endogenous DHEA sulfotransferase expression, especially in liver and intestine (Echchgadda et al. 2004).

Cross sectional studies report on association of lower vitamin D levels with insulinoresistance, accompanying various metabolic states such as diabetes mellitus type 2 (DM2), metabolic syndrome, PCOS and other. This association has been found even in healthy subjects, as shown e.g. in a large Canadian study (Badawi et al. 2014). As the relationships among AIT, glucoregulation or insulinoresistance and vitamin D levels are concerned, we have not found more detailed information in the available literature. Data exist only on the relationship between AIT and insulinoresistance, without any information about vitamin D levels. In the follow up of glycemia, insulinemia or HOMA IR the authors did not find differences between AIT and controls (Biyikli et al. 2014, Mazaheri et al. 2014). However, in patients with AIT with high anti TPO titres (more than $1000 \mathrm{IU} / \mathrm{ml}$ ), higher basal insulinemia was found (Mazaheri et al. 2014).

The performed correlation analysis showed that even in women with AIT significant relationships could be proven, especially under deep vitamin D deficiency. Significant inverse relationships were found between the degree of vitamin D deficiency and markers of insulin secretion (insulin, C peptide) as well as with its efficacy (HOMA IR).

For explanation of this inverse relationship an association of vitamin $\mathrm{D}$ deficiency with low grade inflammation is also emphasized. A really marked positive correlation between levels of $\mathrm{C}$ peptide and insulin was found to an inflammation marker - CRP.

To illustrate the pathological impact of a positive relationship between CRP (according to some authors the best inflammatory marker, Kao 2006) with C peptide and insulin in our vitamin D deficient women with AIT, an inverse relationship of CRP to sex hormone binding globulin (SHBG) levels should be mentioned here. SHBG is believed an important marker of insulinoresistance, its low levels as well as its decreased gene expression are associated with hyperinsulinemia, and after insulinoresistance compensation its levels increase (Winters et al. 2014). It fully agrees with our results showing an inverse negative relationship between SHBG and markers of glucose metabolism (HOMA IR, $C$ peptide and insulin levels) found under vitamin D deficiency.

After correction of vitamin D deficiency the relationships between vitamin $\mathrm{D}$ and parameters of glucose metabolism disappeared, instead of that an inverse relationship of vitamin $\mathrm{D}$ to hepatic insulin resistance (insulin/C peptide) appeared, pointing to its decrease at more pronounced vitamin D supplementation. This relationship corresponds to conclusion of Leung (2016), according to which adequate vitamin D status may modulate hepatic insulin resistance in DM2.

As may be seen from Table 3, as compared with the situation before correction of vitamin D deficiency, new significant relationships appeared between parameters of glucose metabolism and inflammatory markers. Besides already described "deficient" correlation of CRP to insulin secretion the correlation analysis revealed also the relationship to markers of insulin resistance (HOMA IR as well as to hepatic insulin resistance (insulin/C peptide). Of a particular importance we consider newly appearing analogical positive correlations with the marker of thyroid autoimmunity anti TPO. In addition let us mention a positive relationship of another marker of thyroid autoimmunity anti Tg to C peptide.

Supplementation with vitamin D also emphasized the difference of relationships of hepatic insulin resistance (insulin/C peptide) from other parameters of glucose metabolism. In Table 3 we may see that under vitamin D deficient state the only insulin/ $C$ peptide ratio together with glycemia did not correlate with vitamin D. After supplementation this differences were even more pronounced: out of all parameters of glucose metabolism, only insulin/C peptide correlated positively with adrenal androgens (DHEAS as well as with DHEA), and was the only one negatively correlated with vitamin D. Liver is a key organ of metabolic homeostasis, regulating glucose uptake-storageproduction, and it is the key target for insulin action. Hepatic insulin resistance leads to dysregulated glucose metabolism, resulting in hyperglycemia and disturbed interaction with lipogenesis, proteosynthesis and many 
other hepatic functions (Leung 2016, Bechmann et al. 2012, Wallace et al. 2016). These processes in hepatocytes are an object of complicated regulations, studied on molecular-biological level. Our results stress importance of interaction of adrenal androgens with vitamin D in these regulations, especially in influencing hepatic insulin resistance and are an impulse for further clinical and experimental research.

\section{Conclusions}

In conclusion we may summarize: a correlation analysis proved remarkable differences in mutual relationships among vitamin D levels, inflammatory markers, insulin secretion and its efficacy, levels of adrenal androgens and SHBG, under vitamin D deficiency and after its supplementation.

The results opened a number of questions, especially about the relationship between vitamin D and inflammation. Primarily, new mutual relationships appeared after vitamin D supplementation, namely between CRP and a marker of thyroid autoimmunity anti TPO, and the same correlations of both markers to total and hepatic insulin resistance and also to insulin secretion, indicating that increasing $25(\mathrm{OH}) \mathrm{D}$ levels may also be related to proinflammatory states (Mellenthin et al. 2014). A correlation analysis also pointed to a changing role of adrenal androgens in relation to vitamin D, especially in relation to hepatic insulin resistance. The results in the context of existing knowledge may stimulate a discussion concerning vitamin D deficiency in other diseases as well.

\section{Conflict of Interest}

There is no conflict of interest.

\section{Acknowledgements}

The study was supported by the MEYS CR (OP RDE, Excellent research - ENDO.CZ) and by $\mathrm{MH} \mathrm{CZ} \mathrm{-} \mathrm{DRO}$ (Institute of Endocrinology - EÚ, 00023761).

\section{References}

AZIZIEH F, ALYAHYA KO, RAGHUPATHY R: Association between levels of vitamin D and inflammatory markers in healthy women. J Inflamm Res 27: 51-57, 2016.

BADAWI A, SAYEGH S, SADOUN E, AL-THANI M, ARORA P, HADDAD PS: Relationship between insulin resistance and plasma vitamin D in adults. Diabetes Metab Syndr Obes 7: 297-303, 2014.

BECHMANN LP, HANNIVOORT RA, GERKEN G, HOTAMISLIGIL GS, TRAUNER M, CANBAY A: The interaction of hepatic lipid and glucose metabolism in liver diseases. J Hepatol 56: 952-964, 2012.

BIYIKLI HH, ARDUC A, ISIK A, OZUGUZ U, CANER S, DOGRU F, SHORBAGI AI, ERDEN G, BERKER D, GULER S: Assessing the relationship between serum ghrelin levels and metabolic parameters and autoimmunity in patients with euthyroid Hashimoto's thyroiditis. Endocr Pract 20: 818-824, 2014.

CANNELL JJ, GRANT WB, HOLLICK MF: Vitamin D and inflammation. Dermatoendocrinol 29: 983-401, 2015.

CHAUDHARY S, DUTTA D, KUMAR M, SAHA S, MONDAL SA, KUMAR A, MUKHOPADHYAY S: Vitamin D supplementation reduces thyroid peroxidase antibody levels in patients with autoimmune thyroid disease: an open-labeled randomized controlled trial. Indian J Endocrinol Metab 20: 391-398, 2016.

ECHCHGADDA I, SONG CS, ROY AK, CHATTER B: Dehydroepiadrosterone sulfotransferase is a target for transcriptional induction by the vitamin D receptor. Mol Pharmacol 65: 720-729, 2004.

KAO PC, SHIESH SC, WU TJ: Serum reactive protein as a marker for wellness assessment. Ann Clin Lab Sci 36: 163-169, 2006.

KRUIT A, ZANEN P: The association between vitamin D and C-reactive protein levels in patients with inflammatory and non-inflammatory diseases. Clin Biochem 49: 534-537, 2016.

KRYSIAK R, KOWALCZE K, OKOPIEN B: The effect of vitamin D on thyroid autoimmunity in non-lactating women with postpartum thyroiditis. Eur J Clin Nutr 70: 637-639, 2016.

KRYSIAK R, SZKROBKA W, OKOPIEN B: The effect of vitamin D on thyroid autoimmunity in levothyroxinetreated women with Hashimoto's thyroiditis and normal vitamin D status. Exp Clin Endocrinol Diabetes 125 : 229-233, 2017.

LEUNG PS: The potential protective action of vitamin D in hepatic insulin resistance and pancreatic islet dysfunction in type 2 diabetes mellitus. Nutrients 8: 147-164, 2016. 
LIEFAARD MC, LIGTHART S, VITEZOVA A, HOFMAN A, UITTERLINDEN AG, KIEFTE-DE JONG JC, FRANCO OH, ZILLIKENS MC, DEHGHAN A: Vitamin D and C-reactive protein: a mendelian randomization study. PLoS One 10: e0131740, 2015.

MAZAHERI T, SHARIFI F, KAMALI K: Insulin resistance in hypothyroid patients under levothyroxine therapy, a comparison between those with and without thyroid autoimmunity. J Diabetes Metab Disord 13: 103-111, 2014.

MELLENTHIN L, WALLSCHOFSKI H, GROTEVENDT A, VOLZKE H, NAUCK M, HANNEMANN A: Association between serum vitamin D concentrations and inflammatory markers in the general adult population. Metabolism 63: 1056-1062, 2014.

OTT J, PECNIK P, PROMBERGER R, PILS S, SEEMANN R, HERMANN M, FRIGO P: Dehydroepiandrosterone in women with premature ovarian failure and Hashimoto thyroiditis. Climacteric 17: 92-96, 2014.

RUNGE-MORRIS M, KOCAREK TA, FALANY CN: Regulation of the cytosolic sulfotransferases by nuclear receptors. Drug Metab Rev 45: 15-33, 2013.

SOLERTE SB, PRECERUTTI S, GAZZARUSO C, LOCATELLI E, ZAMBONI M, SCHIFINO N, BONACASA R, RONDANELLI M, TACCANI D, FERRARI E, FIORAVANTI M: Defect of a subpopulation of natural killer immune cells in Graves' disease and Hashimoto's thyroiditis: normalizing effect of dehydroepiandrosterone sulfate. Eur J Endocrinol 152: 703-712, 2005.

TAUBNER K, SCHUBERT G, PULZER F, PFAEFFlE R, KOERNER A, DIETZ A, THIERY J, KIESS W, KRATZSCH J: Serum concentrations of anti-thyroid peroxidase and anti-thyroglobulin antibodies in children and adolescents without apparent thyroid disorders. Clin Biochem 47: 3-7, 2014.

VONDRA K, STARKA L, HAMPL R: Vitamin D and thyroid diseases. Physiol Res 64 (Suppl 2): S95-S100, 2015.

WALLACE IR, WALLACE HJ, MCKINLEY MC, BELL PM, HUNTER SJ: Vitamin D and insulin resistance. Clin Endocrinol (Oxf) 84: 159-171, 2016.

WINTERS SJ, GOGINENI J, KAREGAR M, SCOGGINS C, WUNDERLICH CA, BAUMGARTNER R, GHOORAY DT: Sex hormone-binding globulin gene expression and insulin resistance. J Clin Endocrinol Metab 99: E2780-E2788, 2014.

WISSER TJ: Pathways of thyroid hormone metabolism. Acta Med Austriaca 23: 10-16, 1996.

ZHAO D, OUYANG P, DE BOER IH, LUTSEY PL, FARAG YM, GUALLAR E, SISCOVICK DS, POST WS, KALYANI RR, BILLUPS KL, MICHOS ED: Serum vitamin D and sex hormones levels in men and women: The Multi-Ethnic Study of Atherosclerosis (MESA). Maturitas 96: 95-102, 2017. 